\title{
The structure of a hydromagnetic front in a cold collision-free plasma
}

\author{
By P. G. SAFFMAN \\ King's College, London \\ (Received 23 June 1961)
}

\begin{abstract}
A one-dimensional steady solution of the equations of motion of a cold plasma in a magnetic field is obtained. The plasma is of semi-infinite extent, bounded by a plane interface which separates it from a vacuum or medium at rest. The particles approach from infinity, are reflected at the front, and return to infinity in the opposite direction. At infinity, the magnetic field is parallel and anti-parallel to the plasma streams, and is inclined at an angle to the normal to the interface. The front is a current sheet across which the lines of force are bent, with the component of the magnetic field in the plane of the front changing direction. The inertia of the electrons is neglected, and the characteristic frequency associated with the front is the ion gyro-frequency.
\end{abstract}

\section{Introduction}

Interest in the existence and properties of shock waves in a rare ionized gas in the presence of a magnetic field has led in recent years to a study of finite amplitude hydromagnetic waves in a cold collision-free plasma (Ferraro 1955; Adlam \& Allen 1958; Davis, Lüst \& Schlüter 1958; Montgomery 1959; Saffman $1961 a, b)$. The term hydromagnetic is used here for wave motions in which the charge separation is negligible and the plasma may be assumed to be electrically neutral. The solutions so far obtained are of different types; infinite wave trains, solitary waves, and 'quasi-shocks'; but they have one feature in common, namely, that they are all one-dimensional waves in a plasma of infinite extent.

Finite amplitude motions in a plasma of semi-infinite extent bounded by a magnetic field have been obtained by Rosenbluth \& Garwin (1954). $\dagger$ In these flows, the particles approach from infinity, are reflected at the magnetic wall, and return to infinity. The direction of the magnetic field is perpendicular to the particle paths, and the magnetic field becomes zero at infinity. The thickness of the front or sheath is controlled by the electrons which alone are affected by the magnetic field, the ions moving in straight lines and being affected by the electric field only.

In the present note, we shall demonstrate the existence of further finiteamplitude motions in a plasma of semi-infinite extent bounded by a plane front, the space on the other side of the front being either a vacuum or occupied by a neutral gas at rest relative to the front. In these solutions, the flow is steady

$\dagger$ This paper is unpublished, but is described briefly by Mjolsness, Ribe \& Riesenfeld (1961). 
relative to the front, and at a large distance from the front consists of a stream of plasma towards the front and an equal stream away from it in the opposite direction. At infinity, the magnetic field is inclined at an acute angle $\left(<\frac{1}{2} \pi\right)$ to the front, and in the absence of an electric field (corresponding to a suitable velocity of the frame of reference along the front), the streams are parallel to the magnetic field. An individual particle, ion or electron, approaches the front from infinity, turns round at the front, and then returns to infinity. The particles are not brought completely to rest at the front, only the velocity normal to the front being reduced to zero, so that the front is in effect a current sheet. The lines of force are bent considerably on passing through the front and in the vacuum are inclined at an angle of less than $20^{\circ}$ to the front with the direction of the magnetic field component parallel to the front being reversed.

The thickness and structure of the front is now determined principally by the ions, since these have much greater mass and momentum than the electrons and are now affected by the magnetic field. In the following analysis, we shall therefore neglect the mass or inertia of the electrons, and assume that they move in a way such that the electromagnetic forces on them are always in equilibrium. As far as can be seen at present, this seems to be necessary if an exact solution of the non-linear equations of motion is to be obtained; except that a similar exact solution also exists for the special case of equal electron and ion masses, as will be shown briefly in the Appendix.

Referred to a frame of reference moving with either of the streams at infinity, an observer would see a front either advancing into or retreating from a vacuum, and this type of motion might possibly have relevance for certain astrophysical studies or problems concerned with the control and heating of a plasma in the laboratory. However, the physical significance of these fronts is open to doubt for two reasons. First, collisions may prevent the system of two interpenetrating streams at infinity from being a steady state, and secondly, the streams are known theoretically to be unstable to electrostatic plasma oscillations (Kahn 1957; Penrose 1960). Our main purpose here, therefore, is to show that the equations of motion of a cold collision-free plasma admit of a curious type of solution, which may perhaps increase the understanding of plasma dynamics. On the other hand, Dr F. D. Kahn has suggested that even though the configuration may be prevented from being set up exactly as described below, it may exist in a modified form. A brief qualitative description of this is given in $\S 4$, together with another speculation of possible physical interest.

\section{The equations of motion}

We use a frame of reference moving with the front so that the motion is steady. We take the plane of the front as the plane $x=0$. The motion is taken to be onedimensional and uniform in planes parallel to the front. The plasma occupies the region $x<0$, and at $x=-\infty$ there are two uniform streams moving in opposite directions. All thermal motions are neglected.

The suffix $i$ will refer to ions and the suffix $e$ to electrons. The suffix + will refer to quantities in the forward stream moving towards the front, and the suffix - to the backward stream moving away from the front. Thus, $\mathbf{u}_{i+}=\left(u_{i+}, v_{i+}, w_{i+}\right)$ and 
$n_{i+}$ denote the velocity and number density, respectively, of ions in the forward stream for which $u_{i+}>0$. In the backward stream, $u_{i-}<0$. All variables are functions only of $x$.

The equations of motion of the ions and electrons are (Gaussian units are employed)

$$
\begin{aligned}
& m_{i} u_{i_{ \pm}}\left(d \mathbf{u}_{i \pm} / d x\right)=e\left(\mathbf{E}+c^{-1} \mathbf{u}_{i \pm} \wedge \mathbf{H}\right), \\
& m_{e} u_{e \pm}\left(d \mathbf{u}_{\ell \pm} / d x\right)=-e\left(\mathbf{E}+c^{-1} \mathbf{u}_{e \pm} \wedge \mathbf{H}\right),
\end{aligned}
$$

where $m_{i}$ and $m_{e}$ are the masses of the ions and electrons, and the usual notation is employed for the electromagnetic quantities. We suppose that at infinity each stream is uniform and electrically neutral, so that the equations of continuity for the streams are

$$
n_{i+} u_{i+}=n_{e+} u_{e+}=-n_{i-} u_{i-}=-n_{e-} u_{e--}=\text { const., }
$$

provided the front is not a source or sink of charged particles.

With the present geometry, Maxwell's equations reduce to $\mathbf{E}=(E(x), 0,0)$, $H_{x}=$ constant $=H_{0}$, say, and curl $\mathbf{H}=4 \pi \mathbf{j} / c$, where the current density is

$$
\mathbf{j}=e\left(n_{i+} \mathbf{u}_{i+}+n_{i-} \mathbf{u}_{i-}-n_{e+} \mathbf{u}_{e+}-n_{e-} \mathbf{u}_{e-}\right) .
$$

The quasi-neutral approximation (employed in place of the Maxwell equation for the divergence of the electric field) states that

$$
n_{i+}+n_{i-}=n_{e+}+n_{e-} \text {. }
$$

Let us now suppose that at infinity the magnetic field is inclined at an angle $\alpha$ to the $x$-axis, i.e. the direction normal to the front, and lies in the $(x, y)$-plane. That is, we take the components of the magnetic field at infinity to be $\left(H_{0}, H_{0} \tan \alpha, 0\right)$. The velocity of the particles in the forward stream has components $(U, U \tan \alpha, 0)$, where $U$ denotes the velocity component of the stream normal to the front. The general solution of the equations is a difficult matter, and the present analysis will be devoted to obtaining a particular class of symmetrical solutions in which the number densities of the two streams are everywhere equal and the $z$-component of the magnetic field is identically zero. The latter condition requires that the current is parallel to the $z$-axis. The former condition implies, by virtue of $(2.3)$ and (2.5), that

and

$$
\left.\begin{array}{l}
n_{i+}=n_{i-}=n_{e+}=n_{e-}=n, \text { say } \\
u_{i+}=-u_{i-}=u_{e+}=-u_{e-}=u, \text { say; }
\end{array}\right\}
$$

that is, the magnitudes of the velocity component normal to the front is the same in the two streams. Further,

$$
n u=N U,
$$

where $N$ is the number density of ions or electrons in each stream at infinity.

It can now be seen by inspection that if

$$
\mathbf{u}_{i+}=\left(u, v_{i}, w_{i}\right) \text { and } \mathbf{u}_{e+}=\left(u, v_{e}, w_{e}\right)
$$

are solutions of (2.1) with $\mathbf{E}=(E, 0,0)$ and $\mathbf{H}=\left(H_{0}, H_{y}, 0\right)$, then

$$
\mathbf{u}_{i-}=\left(-u,-v_{i}, w_{i}\right) \text { and } \mathbf{u}_{e-}=\left(-u,-v_{e}, w_{e}\right)
$$


are likewise solutions. Moreover, we now have

so that $H_{z} \equiv 0$ and

$$
\begin{gathered}
\mathbf{j}=\left\{0,0,2 n e\left(w_{i}-w_{e}\right)\right\}, \\
d H_{y} / d x=8 \pi n e c^{-1}\left(w_{i}-w_{e}\right) .
\end{gathered}
$$

The relationship between the velocities in the two streams is that the velocities in the plane containing the magnetic field and the normal to the front are equal and opposite, whereas the velocities in the direction perpendicular to the field and parallel to the front are the same.

The boundary conditions to be satisfied on the front $x=0$ are that $u=0$ and also that $v_{i}=v_{e}=0$, since in these symmetrical solutions the $y$-component of the particle velocity is reversed and the motion of an individual particle must be continuous.

\section{An exact solution for the case $m_{e}=0$}

We shall now suppose that the mass of the electrons is negligible, and put $m_{\boldsymbol{e}}=0$. As mentioned previously, this approximation is reasonable in view of the fact that the ions are in reality much more massive than the electrons, and the thickness of the front is determined by the inertia of the ions. Then the equation of motion of the electrons, (2.2), reduces to

$$
w_{e}=0, \quad E=0, \quad v_{e}=u H_{y} / H_{0} .
$$

The equation of motion of the ions now has components

$$
\begin{gathered}
m_{i} u(d u / d x)=-e c^{-1} w_{i} H_{y}, \\
m_{i} u\left(d v_{i} / d x\right)=e c^{-1} w_{i} H_{0}, \\
m_{i} u\left(d w_{i} / d x\right)=e c^{-1}\left(u H_{y}-v_{i} H_{0}\right) .
\end{gathered}
$$

The Maxwell equation (2.8) becomes, on multiplying by $u$ and using (2.7),

$$
u\left(d H_{y} / d x\right)=8 \pi N U e c^{-1} w_{i} .
$$

This system of equations has three first integrals. From (3.2) and (3.5), we obtain

$$
u+\left(H_{y}^{2} / 16 \pi N U m_{i}\right)=U+\left(H_{0}^{2} \tan ^{2} \alpha / 16 \pi N U m_{i}\right),
$$

where the conditions $u \rightarrow U, H_{y} \rightarrow H_{0} \tan \alpha$ as $x \rightarrow-\infty$ have been used to fix the arbitrary constant. From (3.3) and (3.5) we have

$$
v_{i}-\left(H_{0} H_{y} / 8 \pi N U m_{i}\right)=U \tan \alpha-\left(H_{0}^{2} \tan \alpha / 8 \pi N U m_{i}\right)
$$

Multiplying (3.2), (3.3) and (3.4) by $u, v_{i}$ and $w_{i}$, respectively, and adding, we obtain

$$
u^{2}+v_{i}^{2}+w_{i}^{2}=U^{2} \sec ^{2} \alpha \text {. }
$$

It is convenient now to introduce an Alfvén velocity and an Alfvén Mach number by the relations

$$
a=\left(H_{0}^{2} / 4 \pi N m_{i}\right)^{\frac{1}{2}} \text { and } \quad M=U / a .
$$

On the front $x=0, u$ and $v_{i}$ must both vanish, and it then follows from the requirement that (3.6) and (3.7) are consistent that either $U=0$ (which is trivial) or

$$
U \sin \alpha / a=1 \text {. }
$$


(The requirement that $v_{e}=0$ on the front is automatically satisfied by (3.1).) We see from (3.10) that, for the particular class of wave motions being considered, the magnetic field at infinity cannot be normal to the front except in the limit of infinite $M$, and also that $M$ must be greater than one.

The value of the transverse magnetic field on the front is

$$
H_{v}(x=0)=-H_{0} \tan \alpha\left(2 M^{2}-1\right)=-H_{0}\left(2 M^{2}-1\right) /\left(M^{2}-1\right)^{\frac{1}{2}},
$$

and this will be the value ahead of the front in the region $x>0$. The direction of the transverse field therefore changes across the front. Also, the strength of the field behind the front (i.e. in the plasma) is weaker than the strength ahead.

We can now determine the structure of the front. We express $u$ in terms of $v_{i}$ by (3.6) and (3.7), and substitute into (3.8) to obtain after a little reduction

by (3.2).

$$
\begin{aligned}
w_{i}^{2} & =\left(v_{i}-U \tan \alpha\right)^{2}\left[\frac{2 M^{2}-3}{\cos ^{2} \alpha}-2 M^{2} \tan \alpha\left(\frac{v_{i}}{U}-\tan \alpha\right)+M^{4}\left(\frac{v_{i}}{U}-\tan \alpha\right)^{2}\right] \\
& =\left(\frac{m_{i} c}{e H_{0}}\right)^{2}\left(u \frac{d v_{i}}{d x}\right)^{2},
\end{aligned}
$$

It follows from (3.12) that for a solution to exist, it is necessary that $2 M^{2}>3$, i.e. $M>\sqrt{\frac{3}{2}}$. This imposes a lower limit on $M$, and from (3.10) an upper limit on $\alpha$, namely $\sin \alpha<\sqrt{\frac{2}{3}}$, i.e. $\alpha<55^{\circ}$. If $\beta$ denotes the angle between the magnetic field ahead of the front and the normal to the wave front, then

$$
\tan \beta=\left(2 M^{2}-1\right) /\left(M^{2}-1\right)^{\frac{1}{2}}>2 \sqrt{ } 2 \text {, i.e. } \beta>70^{\circ} .
$$

Thus the angle between the front and the magnetic field ahead of it will be less than approximately $20^{\circ}$, which is quite small. As $\alpha$ decreases and $M$ increases, the angle $\beta$ increases.

To integrate (3.12), we introduce a dimensionless time $t$ by the relation

$$
\omega_{i}(d x / d t)=u \text {, where } t=0 \text { when } \quad x=0 .
$$

Here, $\omega_{i}=e H_{0} / m_{i} c$ is the gyro-frequency of the ions about the field normal to the front. Then the integral of $(3.12)$ is

where

$$
v_{i}=U \tan \alpha-\frac{2 M^{2}-3}{\left(M^{2}-1\right)\left(2 M^{2}-1\right)^{\frac{1}{2}} \cosh (\lambda t+\epsilon)-\left(M^{2}-1\right)^{\frac{1}{2}}},
$$

$$
\lambda=M\left[\left(2 M^{2}-3\right) /\left(M^{2}-1\right)\right]^{\frac{1}{2}} \quad \text { and } \quad \cosh \epsilon=2\left[\left(M^{2}-1\right) /\left(2 M^{2}-1\right)\right]^{\frac{1}{2}} \quad(\epsilon<0) .
$$

The values of all the other quantities as functions of $t$ now follow from the first integrals of the equations, and the relation between $x$ and $t$ is obtained by integrating (3.14). The full expressions are long and complicated, and will not be given here. We shall just examine the nature of the solution in the neighbourhood of $x=0$.

It is found that

Thus

$$
v_{i} \doteqdot-U t \sec \alpha, \quad u \doteqdot-U t M\left(2 M^{2}-1\right) /\left(M^{2}-1\right) .
$$


and hence $u$ and $v_{i}$ (and $v_{e}$ ) vanish like $(-x)^{\frac{1}{2}}$ on the front. Also,

$$
w_{i}=-U \sec \alpha \text { on } x=0,
$$

so the ions are moving parallel to the negative $z$-axis on the front, and do not come altogether to rest, as do the electrons since $w_{e} \equiv 0$ when $m_{e}=0$. The current is in fact carried entirely by the ions.

The number density $n$ becomes infinite on the front like $(-x)^{-\frac{1}{2}}$. This infinity is integrable, and arises, in fact, from the idealization of taking the plasma to be cold. Of course, the increase in number density at the front implies that collisions may become important, but the possible effect of collisions is not clear.

The quasi-neutral approximation is exact for the above solution since $\mathbf{E}=\mathbf{0}$, and the solution does in fact satisfy the Maxwell equation $\operatorname{div} \mathbf{E}=4 \pi e\left(n_{i}-n_{e}\right)$.

The path of an individual electron is parallel to the $(x, y)$-plane. The ions, however, have a net drift in the negative $z$-direction. The total drift is found easily from (3.3) and is

$$
\frac{2}{\omega_{i}} \int_{-\infty}^{0} w_{i} d t=\frac{2}{\omega_{i}} \int_{-\infty}^{0} \frac{d v_{i}}{d t} d t=-\frac{2 U \tan \alpha}{\omega_{i}}
$$

A condition for the neglect of the electrons' inertia to be valid can be obtained. The approximate equations of motion of the ions are valid if $w_{e} \ll w_{i}$. Now

$$
w_{e}=-\frac{m_{e}}{m_{i}} \frac{d v_{e}}{d t} \div-\frac{m_{e}}{m_{i}} \frac{d}{d t}\left(\frac{u H_{y}}{H_{0}}\right) \text {. }
$$

Substituting for $u$ and $H_{y}$ from the approximate solution, we find that the approximation is self-consistent if $M^{2}\left(m_{e} / m_{i}\right) \ll 1$. Thus the solution breaks down if the Alfvén Mach number is too large.

\section{Discussion}

The hydromagnetic plasma front is unlikely to occur in reality exactly as described, because of the effect of collisions between the particles in the two opposing streams at infinity. However, the mean free path for close collisions of charged particles may be so large that this may not be too serious a difficulty. A more serious objection is the theoretical result (Kahn 1957) that the relative motion of two cold plasma streams is unstable to electrostatic space-charge plasma oscillations, whose frequency will be much larger than the ion gyrofrequency.

However, Dr F. D. Kahn has suggested that this difficulty may be circumvented. The essence of his argument is as follows. The current in the front is carried by the ions, and the role of the electrons is essentially passive. Suppose therefore that the electron gas is not cold, but has at infinity a Maxwellian velocity distribution with a velocity dispersion of the order of $U$, the speed of approach of the ions towards the front. This should not affect the current distribution and the motion of the ions significantly, so that the gross features of the front are unaltered. If now the incident and reflected ion streams have small internal velocity dispersions, then the plasma will be stable against space-charge waves. It follows from Penrose's (1960) work that the velocity dispersion of the ions need only be of the order of $U\left(m_{e} / m_{i}\right)^{\frac{1}{2}}$ to achieve stability. 
Finally, a speculation of possible interest may be in order. The question is: does a flow with a sharpish front and with the ions in relative motion, of the type obtained in this paper, develop if a semi-infinite stream of neutral plasma moves across a magnetic field? Analogous problems occur in several branches of fluid mechanics. For instance, when a gas flows through a tube at a supersonic speed, a flow with shock waves is a theoretical possibility, and it is found in practice that shock waves often occur. Another example is the appearance of hydraulic jumps. If the answer in the present case is in the affirmative, this may possibly provide a means of raising the ion temperature in a plasma.

\section{Appendix. Exact solution for the case $m_{i}=m_{c}$}

With the notation of $\S 2$, it can be seen by inspection that when $m_{i}=m_{e}$, there is a symmetrical solution with

$$
\left.\begin{array}{r}
n_{i+}=n_{e+}=n_{i-}=n_{e-}=n, \text { say; } \\
u_{i+}=u_{e+}=-u_{i-}=-u_{e-}=u, \text { say; } \\
v_{i+}=v_{e+}=-v_{i-}=-v_{e-}=v, \text { say; } \\
w_{i+}=-w_{e+}=w_{i-}=-w_{e-}=w, \text { say. }
\end{array}\right\}
$$

The equations corresponding to (3.2) to (3.5) are now

$$
\begin{aligned}
m_{i} u(d u / d x) & =-e c^{-1} w H_{y}, \\
m_{i} u(d v / d x) & =e c^{-1} w H_{0}, \\
m_{i} u(d w / d x) & =e c^{-1}\left(u H_{y}-v H_{0}\right), \\
u\left(d H_{y} / d x\right) & =16 \pi N U e c^{-1} w .
\end{aligned}
$$

These equations are exactly the same as the former, except for a factor 2 in (A5) as compared with (3.5). If we now take as an Alfvén velocity and Alfvén Mach number,

$$
a^{\prime}=\left\{H_{0}^{2} / 4 \pi N\left(m_{i}+m_{e}\right)\right\}^{\frac{1}{2}}, \quad M^{\prime}=U / a^{\prime},
$$

then the previous solution of $\S 3$ holds exactly with $M$ replaced by $M^{\prime}$.

\section{REFERENCES}

Adlam, J. H. \& Allen, J. E. 1958 Phil. Mag. 3, 448.

DAvis, L., Lüst, R. \& SChLÜTER, A. $1958 Z$. Naturforsch. $13 a, 916$.

Ferraro, V. C. A. 1955 Proc. Roy. Soc. A, 233, 310.

KAHN, F. D. 1957 J. Fluid Mech. 2, 601.

MJolsness, R. C., Ribe, F. L. \& RIEsenfeld, W. B. 1961 Phys. Fluids, 4, 730.

Montgomery, D. 1959 Phys. Fluids, 2, 585.

Pennose, O. 1960 Phys. Fluids, 3, 258.

Rosenbluth, M. \& Garwin, R. 1954 Los Alamos Report LA-1850 (unpublished).

SAFFMAN, P. G. 1961 a J. Fluid Mech. 11, 16.

SAfmMan, P. G. $1961 b$ J. Fluid Mech. 11, 552. 